\title{
Metabolome and transcriptome profiling reveals that terpenoid biosynthesis dominates the growth and development of Sanghuangporus baumii
}

\author{
Zengcai Liu ${ }^{1}$, Shixin Wang ${ }^{1}$, Ruipeng Liu ${ }^{1}$, Xinyu Tong ${ }^{1}$, Yawei Li ${ }^{1}$, Xutong Wang ${ }^{1}$, and Li \\ $\mathrm{Zou}^{1}$ \\ ${ }^{1}$ Northeast Forestry University School of Forestry
}

January 31, 2022

\begin{abstract}
Sanghuangporus baumii is a traditional medicinal fungus that produces pharmacological terpenoids, but natural resources are insufficient for applications, and growth and development mechanisms are poorly understood. Combining metabolomic and transcriptomic analyses, we identified four terpenoid hormones and a central gene isopentenyl diphosphate isomerase (IDI) involved in growth and development. The importance of the four terpenoid hormones was further confirmed by exogenous hormones addition, we found that Cis-Zeatin (CZ) plays a major role in the mycelia stage, Trans-Zeatin (TZ) and Gibberellin A4 (GA4) are important in the primordia stage, GA4 is crucial for the fruiting bodies stage, and Abscisic Acid (ABA) may be a marker of maturity. Later, hormone content determination and qRT-PCR were used to verify the growth and development mechanism, and finally it was confirmed that IDI gene affects the content of terpenoid hormones content by regulating the transcript level of terpenoid biosynthesis genes, thus affecting the morphological changes of S. baumii. Our results illuminate the growth and development mechanisms of $S$. baumii, and may promote the breeding and utilisation of high-quality varieties.
\end{abstract}

Metabolome and transcriptome profiling reveals that terpenoid biosynthesis dominates the growth and development ofSanghuangporus baumii

Zengcai Liu, Shixin Wang, Ruipeng Liu, Xinyu Tong, Yawei Li, Xutong Wang, Li Zou*

College of Forestry, Northeast Forestry University, Harbin, Heilongjiang, China.

* Corresponding author: Li Zou

Email: 13903650896@163.com

Tel /Fax: +86-0451-86660457

Funding information

Fundamental Research Funds for the Central Universities of China, Grant/Award Number: 2572018AA34; National Natural Science Foundation of China, Grant/Award Number: 32171792.

\section{Abstract}

Sanghuangporus baumii is a traditional medicinal fungus that produces pharmacological terpenoids, but natural resources are insufficient for applications, and growth and development mechanisms are poorly understood. Combining metabolomic and transcriptomic analyses, we identified four terpenoid hormones and a central gene isopentenyl diphosphate isomerase (IDI) involved in growth and development. The importance of the four terpenoid hormones was further confirmed by exogenous hormones addition, we 
found that Cis-Zeatin (CZ) plays a major role in the mycelia stage, Trans-Zeatin (TZ) and Gibberellin A4 (GA4) are important in the primordia stage, GA4 is crucial for the fruiting bodies stage, and Abscisic Acid (ABA) may be a marker of maturity. Later, hormone content determination and qRT-PCR were used to verify the growth and development mechanism, and finally it was confirmed that IDI gene affects the content of terpenoid hormones content by regulating the transcript level of terpenoid biosynthesis genes, thus affecting the morphological changes of $S$. baumii . Our results illuminate the growth and development mechanisms of $S$. baumii , and may promote the breeding and utilisation of high-quality varieties.

\section{KEYWORDS}

Sanghuangporus baumii, growth and development, metabolome and transcriptome, isopentenyl diphosphate isomerase, terpenoid biosynthesis

\section{INTRODUCTION}

Sanghuangporus baumii (Pilát) L.W. Zhou \& Y.C. Dai is a precious medicinal fungus with medicinal effects including improving immunity, lowering blood sugar, and anti-cancer and anti-tumour activities (Huang et al., 2020; Zheng et al., 2021). The market demand for $S$. baumii is increasing, and this is gradually exhausting wild resources (Liu, Sun, Wang, \& Zou, 2020). Wild-type (WT) S. baumii is host-specific and grows only on Syringa reticulata. It grows slowly, taking 1-2 years to develop mature fruiting bodies suitable for human use. Due to the scarcity of its $S$. reticulata host in the wild, and the slow growth rate of $S$. baumii, existing $S$. baumii wild resources are being depleted needs, hence domestication and cultivation are being actively explored.

The growth and development process of $S$. baumii involves growth from mycelia (Myc) to primordia (Pri) followed by the formation of fruiting bodies (Fru; Figure 1). However, because the growth and development mechanism of $S$. baumii remains unclear, the yield is unstable and the quality is uneven. As molecular biology technology has developed, metabolome and transcriptome studies are being used to uncover the growth mechanism in greater detail (Qian et al., 2021; Wang et al., 2020). Changes in metabolites and corresponding genes associated with growth and development would be detected during growth and development process (Gong et al., 2021). Therefore, to understand these changes in S. baumii at different developmental stages, metabolome and transcriptome would be of great value.

Terpenoids are important molecules medicinal research, as exemplified by artemisinin (Ro et al., 2006), taxol (Ajikumar et al., 2010) and ganoderic acid (Zhang et al., 2017), and they are also involved in growth and development, including Cis-Zeatin (CZ), Trans-Zeatin (TZ; Kaori et al., 2006), Abscisic Acid (ABA; Tan, Schwartz, Zeevaart, \& McCarty, 1997) and Gibberellin A4 (GA4; Schomburg, Bizzell, Lee, Zeevaart, \& Amasino, 2003). Terpenoids can be categorised into monoterpenoids, sesquiterpenoids, diterpenoids, triterpenoids and steroids (Huang et al., 2020; Liu, Sun, Wang, \& Zou, 2020; Zheng et al., 2021). They are synthesised by the mevalonate (MVA) pathway, and geranyl pyrophosphate synthase (GPS), famesyl pyrophosphate synthase (FPPS), geranylgeranyl pyrophosphate synthase (GGPS), squalene synthase (SQS) and lanosterol synthase (LS) are direct precursor genes for the biosynthesis of monoterpenoids, sesquiterpenoids, diterpenoids, triterpenoids and steroids (Ali et al., 2017). Despite scientific and medicinal interest in the terpenoids synthesis pathway of $S$. baumii, the compounds present and their biosynthesis-related genes have not yet been fully elucidated. Meanwhile, terpenoid hormones have been studied more in terms of growth and development in plants than fungi, so they may also play a distinct role in the growth and development of $S$. baumii .

Understanding the growth and development mechanism and the terpenoids present in $S$. baumii could be helpful for improving yield and quality. In the present work, we collected material from three different developmental stages of WT S. baumii (Myc, Pri and Fru). To clarify the growth and development mechanism, metabolomics and transcriptomics approaches were employed to analyse differences in metabolite abundance and gene expression, and explore correlations between terpenoid biosynthesis and gene expression. A central geneIDI and four terpenoid hormones involved in growth and development were identified. Then we confirmed the importance of the four terpenoid hormones by exogenous hormones addition. To 
further verify the growth and development mechanism of $S$. baumii, we further measured the transcript levels of terpenoid genes and the content of terpenoid metabolites between WT and IDI transformant (IT). Finally, it was confirmed that IDI gene dominates the growth and development of $S$. baumii by regulating the transcript level of terpenoid biosynthesis genes and the content of terpenoid hormones. The results revealed the terpenoids present in S. baumii and the associated terpenoid biosynthesis genes. Additionally, terpenoid hormones related to growth and development, and the biosynthesis pathway were identified. The results will likely promote the cultivation of high-quality $S$. baumii in the future.

\section{MATERIALS AND METHODS}

\subsection{Fungal materials and collection}

WT fruiting bodies (WT Fru) were collected from S. reticulata at Liangshui Nature Reserve, Lesser Xing'an Mountains, Yichun city, Heilongjiang Province, China (Figure 1). Mycelia isolated from Fru were identified as $S$. baumii according to ITS sequence alignment. Mycelia were inoculated in potato dextrose (PD) medium and grown in a shaking incubator at $170 \mathrm{rpm}$ and $25 \mathrm{deg}$ for 10 days, and WT mycelia (WT Myc) were collected (Figure 1). Subsequently, mycelia were inoculated into polypropylene bags containing culture matrix to collect WT primordia (WT Pri; Figure 1). For detailed culture methods and conditions refer to Liu et al (Liu, Sun, Wang, \& Zou, 2020).

\subsection{Widely targeted metabolome analysis}

Three biological replicates were collected for each stage, and all samples were immediately frozen in liquid nitrogen and stored at -80degC. Freeze-dried samples were crushed and extracted following previously reported (Cai, Weng, Guo, Peng, \& Zhu, 2015). QC samples were prepared by mixing all samples to determine the reproducibility of the obtained results. These samples were stored at $-80 \mathrm{deg} C$ until ultrahigh-performance liquid chromatography electrospray ionisation tandem mass spectrometry (UHPLC-MS/MS) analysis.

Quantitative analysis of metabolites was accomplished by multiple reaction monitoring (MRM). SCIEX Analyst Work Station Software (Version 1.6.3) was employed for MRM data acquisition and processing. MS raw data (.wiff) files were converted to TXT format using MSconventer software (Meng et al., 2019). In-house $\mathrm{R}$ programs and databases were applied for peak detection and annotation (Kuhl, Tautenhahn, Bottcher, Larson, \& Neumann, 2012; Zhang et al., 2015). Metabolome analysis was conducted by Guangzhou Magigene Biotechnology Company (Guangzhou, China). Differential metabolites (DEMs) were screened based on $p$ -value $<0.05$ among the metabolites with VIP $>1$.

\subsection{Transcriptome sequencing and data analysis}

For transcriptome sequencing the sampling method was the same as the metabolome analysis experiment, with three biological replicates for each sample. Total RNA was extracted using an RNAiso Plus Kit (Takara, Dalian, China) according to the manufacturer's directions. RNA samples that met the requirements were sent to Magigene Biotechnology Company (Guangzhou, China) for transcriptome sequencing on an Illumina HiSeq 2500 platform.

Raw reads in fastq format were first analysed for quality control. Reads containing adapters, sequences with more than $10 \%$ unknown nucleotides $(\mathrm{N})$, and low-quality reads were primarily removed from the raw dataset. All subsequent analyses were based on the high-quality clean data. All nine libraries (Myc, Pri and Fru, three replicates for each treatment) were compared with the reference genome sequence of S. baumii(https://www.ncbi.nlm.nih.gov/genome/?term=Sanghuangporus\%20baumii), and reads were compared with the relevant reference sequence to determine sequencing saturation. Gene expression levels were calculated as fragments per kilobase per transcript per million mapped reads (FPKM), and principal component analysis (PCA) and gene expression pattern cluster mapping were performed. Genes with FDR [?]0.05 and $\mid \log 2$ (fold change)| [?]1 were designated as differentially expressed genes (DEGs), and those DEGs were annotated using KEGG pathway analysis.

\subsection{Validation of gene expression from transcriptome data}


The Illumina sequencing results were validated by qRT-PCR for 16 DEGs involved in terpenoid biosynthesis. Their primers for qRT-PCR were designed by Primer Premier 5.0 software (Table S1). The S. baumiisamples were the same as those used for transcriptome analysis with three biological replicates for each treatment. qRT-PCR was performed on a CFX 96 real-time PCR detection system (Bio-Rad, California, USA). All protocols were carried out according to the manufacturer's instructions. Relative expression levels of target genes were calculated using the $2^{-\mathrm{Ct}}$ method (Livak \& Schmittgen, 2001) with the $a$-tubulin gene as an internal control.

2.5 Adding or spraying exogenous terpenoid hormones in the growth and development stage of $S$. baumii

Positive IDI transformant (IT) strain of $S$. baumii was obtained in previous (unpublished), IT materials (IT Myc, Pri and Fru) were cultured in accordance with previous reports (Liu, Sun, Wang, \& Zou, 2020). To test the effects of hormones CZ, TZ, ABA and GA4 on the growth and development of (WT and IT) S. baumii , $10 \mathrm{mg} / \mathrm{L} \mathrm{CZ,} \mathrm{TZ,} \mathrm{ABA} \mathrm{and} \mathrm{GA4} \mathrm{were} \mathrm{separately} \mathrm{added} \mathrm{to} \mathrm{PDA} \mathrm{medium.} \mathrm{Myc} \mathrm{and} \mathrm{Pri} \mathrm{of} \mathrm{WT}$ and IT S. baumii samples with a diameter of $0.5 \mathrm{~cm}$ were inoculated on PDA petri dishes, and the growth rate of Myc and the germination rate of Pri samples were observed after 10 days. WT and IT S. baumii Fru were sprayed with the same concentration of four exogenous hormones, three times a day, and the effects of these four hormones on the dry weight of Fru samples were measured after 20 days. All experiments were repeated three times for each group. Finally, the growth rate of Myc, the germination rate of Pri, and the dry weight of Fru were measured.

\subsection{Determination of the transcript level of gene and hormone content}

Myc, Pri and Fru materials from WT and IT S. baumii were collected as described for the metabolome experiment. Gene transcript levels were determined by qRT-PCR as described above. Determination method of hormone content is as follows: A $0.5 \mathrm{~g}$ quantity of each individual sample was weighed, crushed, and mixed with $4.5 \mathrm{~mL}$ of phosphate-buffered saline (PBS) extract solution (pH7.4). After centrifuging at $12,000 \times \mathrm{g}$ for $15 \mathrm{~min}$, the supernatant was carefully transferred to a $10 \mathrm{~mL}$ centrifuge tube. Levels of $\mathrm{CZ}, \mathrm{TZ}, \mathrm{ABA}$ and GA4 were determined using ELISA kits (mlbio, Shanghai, China) following the manufacturer's instructions. The total triterpenoids content was measured as described previously (Liu, Sun, Wang, \& Zou, 2020).

\section{RESULTS AND DISCUSSION}

\subsection{Metabolome analysis of different developmental stages of S. baumii}

A widely targeted metabolome analysis was performed on samples taken from different developmental stages of $S$. baumii , and 452 metabolites were obtained across all samples. In the PCA plot, the QC samples grouped together, indicating that the QC samples had similar metabolic profiles, and that the entire analysis was reliable and repeatable. Biological replicates for each of the three different development stages clustered together in different areas, indicating that there were significant differences in metabolism (Figure 2A). Differential metabolites are illustrated using a Venn diagram (Figure 2B), a bar chart (Figure 2C) and Volcano plots (Figure 2D). There were 51 differentially abundant metabolites (21 upregulated and 30 downregulated) between Fru and Myc. There were 32 differentially abundant metabolites ( 8 upregulated and 24 downregulated) between Fru and Pri. There were 28 differentially abundant metabolites (15 upregulated and 13 downregulated) between Pri and Myc.

\subsection{Terpenoid analysis related to growth and developmentof}

S. baumii

A total of 125 terpenoids were identified across the three different developmental stages, including 2 zeatins, 12 monoterpenoids, 23 sesquiterpenoids, 21 diterpenoids, 28 triterpenoids and 39 steroids (Figure 2E; Table S2). There were four terpenoid hormones related to growth and development, including CZ, TZ, ABA and GA4. ELISA results showed that CZ content was highest in the Myc stage $(1.18 \mathrm{mg} / \mathrm{g})$, TZ and GA4 level were highest in the Pri stage $(1.27 \mathrm{mg} / \mathrm{g}$ and $843.00 \mathrm{pmol} / \mathrm{g})$, and ABA content was highest in the Fru stage 
(896.11 ng/g; Figure S1). Variation in the content of the four terpenoid hormones determined by ELISA was essentially consistent with levels measured by UHPLC-MS/MS, with correlation coefficient R $>0.885$, indicating that ELISA kit determination for all four terpenoid hormones was stable and reliable. These results tentatively suggested that $\mathrm{CZ}$ plays an important role in the Myc stage, while $\mathrm{TZ}$ and GA4 are important in the Pri stage, and ABA is crucial in the Fru stage.

\subsection{Differentially expressed genes between the three different developmental stages of S. baumii}

Three independent replicates for the three different developmental stages of $S$. baumii were subjected to RNASeq analyses. The results showed that the biological replicates for each of the three different development stages clustered together in different areas and the number of detected genes tends to change little with any further increase after reaches a certain value, indicating that there were significant differences in the genes (Figure 3A), and adequate sequencing saturation (Figure S2). Using RNA-Seq technology, 8.09 Gb, 8.59 Gb and $6.74 \mathrm{~Gb}$ of raw data were obtained from samples of the three different developmental stages, and the total number of clean paired reads was $24,633,634,26,032,769$ and 20,39,8644. This resulted in approximately $6.02-7.59 \mathrm{~Gb}$ of clean reads, a Q20 value $>98.64 \%$, a Q30 value $>94.94 \%$, and an average GC \% ratio of $52.08 \%$ (Table S3). It can be seen from Figure S3 that the three replicates for each sample clustered together, indicating the stability and repeatability of the data, and Myc and Pri may reflect growth and development stages and therefore share similar gene expression patterns.

A total of 4075 DEGs (1770 upregulated and 2305 downregulated) were identified between Fru and Myc groups. There were 3860 DEGs (1580 upregulated and 2280 downregulated) between Fru and Pri. There were 2408 DEGs (1216 upregulated and 1192 downregulated) between Pri and Myc (Figure 3B, C). FPKM values of these DEGs were provided in Table S4.

\subsection{Genes related to terpenoid biosynthesis}

Many terpenoids were detected in S. baumii and they were synthesised by the MVA pathway, which were annotated in various KEGG pathways including terpenoid backbone biosynthesis, zeatin biosynthesis, sesquiterpenoid biosynthesis, diterpenoid biosynthesis, and triterpenoid and steroid biosynthesis (Figure 4; Table S5). TZ synthesis genes and ABA synthesis genes were not annotated in zeatin and sesquiterpenoid biosynthesis pathway. Based on previous reports, we found CYP5340A71, CYP5340A72 (named by the P450 naming committee) and ABA4 through homology comparison. The CYP5340A71 and CYP5340A72 genes of $S$. baumiiclustered together with genes AtCYP735A1 and AtCYP735A2 of Arabidopsis (Takei, Yamaya, \& Sakakibara, 2004). The ABA4 gene of $S$. baumii clustered with Botrytis cinerea BcABA4(Gong, Shu, Yang, Ding, \& Tan, 2014). These results (Figure S4) proved that the terpenoid biosynthesis genes used in this study were reliable. Furthermore, ABA is probably synthesised by the MVA pathway in fungi (Gong, Shu, Yang, Ding, \& Tan, 2014), but this needs to be further verified in S. baumii .

Some transcripts expressed at high levels were found to encode terpenoid biosynthesis genes in S. baumii , including HMG-CoA synthase (HMGS), HMG-CoA reductase (HMGR), mevalonate pyrophosphate decarboxylase (MVD), squalene epoxidase (SE), ABA4 and ergosterol C14 reductase (ERG24; Table S6). Our results are similar to those from previous studies on the transcriptomes of Salvia officinalis and S. guaranitica (Ali et al., 2017; Ali et al., 2018). Additionally, some growth and development-related terpenoid synthesis genes were significantly correlated with the abundance patterns of their synthesised products, such as $I D I$ gene and $\mathrm{CZ}, C Y P 5340 A 71$ and $C Y P 5340 A 72$ genes and TZ, the ABA4 gene and ABA, and the GPPS gene and GA4, all showing positive correlations (Figure 3D). This indicates that key genes will play an active role in the biosynthesis of the metabolites (Gong Shu, Yang, Ding, \& Tan, 2014; Schmiderer, Grausgruber-Groger, Grassi, Steinborn, \& Novak, 2010; Takei et al., 2004).

\subsection{Validation of gene expression patterns by qRT-PCR}

To validate the accuracy and repeatability of the transcriptome data, qRT-PCR was conducted on a set of DEGs associated with terpenoid metabolism. Among all candidate DEGs related to terpenoid biosynthesis, 16 genes were selected for qRT-PCR analysis. Figure 5 shows the expression levels of all 16 selected DEGs 
determined by qRT-PCR and RNA-Seq. Overall, the expression profiles of the 16 DEGs as determined by qRT-PCR were consistent with the corresponding FPKM values derived from RNA-Seq analysis. Most genes exhibited a similar expression pattern using both methods $(\mathrm{R}>0.690)$, indicating that the expression data obtained by RNA-Seq were reliable.

We conducted qRT-PCR analyses to confirm the expression patterns of growth and development-related genes in S. baumii. We observed a correlation and linkage between terpenoid products and terpenoid synthase gene expression levels at different stages. For instance, the highest $\mathrm{CZ}$ product abundance and IDI gene expression level were observed in Myc followed by Pri then Fru. Also, we revealed a correlation and linkage between TZ and CYP5340A71 and CYP5340A72 gene expression levels at different developmental stages. Similarly, the highest ABA product abundance and the highest $A B A 4$ gene expression levels were observed in Fru followed by Pri then Myc (Figure 3D, 5). Some of our results are in agreement with those of previous studies (Liu et al., 2021; Takei, Yamaya, \& Sakakibara, 2004) showing that terpenoid quantities are mainly controlled transcriptionally.

\subsection{Effects of four exogenous terpenoid hormones on the growth and development of WT and} IT $S$. baumii strains

The IDI gene shows a significant correlation with the synthesis of $\mathrm{CZ}$, and it is also related to the synthesis of a common precursor of four terpenoid hormones (Figure 3D), hence it was believed to play an important role in the growth and development of $S$. baumii . Fortunately, we obtained an IT strain at the early stage of the experiment, and we found significant morphological characteristics differences between WT and IT (i.e. Myc, Pri and Fru) in the culture process (Figure 6A). To test the metabolome hypothesis and demonstrate the role of these terpenoid hormones, we investigated the effects of adding or spraying four exogenous hormones (CZ, TZ, ABA and GA4) on the growth and development process of WT and IT strains of S. baumii .

The results showed that supplementation each of the four exogenous terpenoid hormones did not significantly affect the growth of WT Myc; rather, there was a slight decrease, possibly indicating that the hormone content in Myc had reached a balance, and there may have even been a negative feedback effect (Figure $6 \mathrm{~B}, \mathrm{C})$, this has also been found in WT Pri and IT Fru groups. In the IT Myc group, only the growth rate of Myc supplemented with CZ was faster than that of IT control Myc. (Figure 6B, C). In WT and IT Pri groups, addition of each of the four exogenous hormones had a significant inhibitory effect on germination rate of WT Pri, while addition of TZ and GA4 promoted the germination rate of IT Pri (Figure 6D, E). In WT and IT Fru groups, only spraying of GA4 promoted the growth of WT Fru, while spraying the other three exogenous hormones had no obvious effect on the growth and development of Fru (Figure 6F, G).

By adding exogenous hormones, we found that $\mathrm{CZ}$ played a positive role in promoting growth of $S$. baumii in the Myc stage, while TZ and GA4 were important in the Pri stage, these results are consistent with the above metabolome results, suggesting phenotypic changes may be influenced by a single hormone, or may be caused by the combined action of two or more hormones (Peter et al., 2020). However, GA4 had a strong influence during the Fru stage, rather than ABA as previously speculated, it needs to be validated and explained next.

\subsection{Differences in gene expression and terpenoid content between WT and IT S. baumii strains}

$I D I$ gene overexpression did not accelerate the growth rate at all stages as expected (Figure 6A). To explore the causes of this phenomenon, we further measured the transcript levels of terpenoid genes (Figure 7A) and the content of terpenoid metabolites (Figure 7B). By measuring the contents of the four terpenoid hormones and the transcript levels of related genes in WT and IT S. baumii, we found that WT Myc grew faster than IT Myc, which be due to a significant increase in the content of CZ, level of which is regulated by corresponding genes. WT Pri grew faster than IT Pri, which may be due to a significant increase in the content of one or two hormones in TZ and GA4, and levels of these hormones are also regulated by corresponding genes. IT Fru grew faster than WT Fru, possibly due an increase in the content of GA4, and level of GA4 is regulated by corresponding genes (Figure 7). 
Overexpression of the IDI gene does not increase the contents of all terpenoid hormones, mainly due to competition among hormones and tissue-specific expression (Barreto, Herken, Silva, MunneBosch, \& Garcia, 2020; Zhang et al., 2014). IT S. baumii CZ, TZ and GA4 levels and related genes were decreased in Myc and Pri stages, which was probably caused by an increase in triterpenoids content caused by high-level expression of triterpenoids biosynthetic genes (Figure 7). When the copy number of triterpenoids biosynthesis genes was low, the triterpenoids content was significantly decreased, and the GA4 content and related genes of GA4 biosynthesis were significantly increased, resulting in IT Fru grew faster than WT Fru (Figure 7). This also explains why GA4 had a strong influence during the Fru stage rather than ABA. ABA seems to be a sign of maturity, and its content is only higher in fast-growing or large tissues (Figure 6A, 7B), this phenomenon was also observed in the growth and development of strawberry (Kim, Lee, Hong, \& Lee, 2019).

\section{CONCLUSION}

Metabolomics and transcriptomics approaches were applied to identify four terpenoid hormones and the associated biosynthesis key geneIDI, which are related to the growth and development of $S$. baumii. The results were further supported by addition of the four exogenous terpenoid hormones. Specifically, CZ played a major role in the Myc stage, TZ and GA4 functioned in the Pri stage, and GA4 influenced the Fru stage. ABA may be a marker of maturity of $S$. baumii . We further measured the transcript levels of terpenoid genes and the content of terpenoid hormones. The underlying mechanism was found that the phenotypic changes were influenced by terpenoid hormones, level of which is regulated by corresponding genes. The findings provide important guidance for molecular breeding of $S$. baumii .

\section{ACKNOWLEDGMENTS}

This work was supported by the Fundamental Research Funds for the Central Universities of China (2572018AA34), the National Natural Science Foundation of China (32171792).

\section{CONFLICT OF INTERESTS}

The authors declare that they have no conflict of interest.

\section{DATA AVAILABILITY}

Supplementary data to this article can be found online at...

\section{REFERENCES}

Ajikumar, P. K., Xiao, W. H., Tyo, K. E. J., Wang, Y., Simeon, F., Leonard, E., .. Stephanopoulos, G. (2010). Isoprenoid pathway optimization for taxol precursor overproduction in Escherichia coli . Science, 330 (6000), 70-74.

Ali, M., Hussain, R. M., Rehman, N. U., She, G. B., Li, P. H., Wan, X. C., .. Zhao, J. (2018). De novo transcriptome sequencing and metabolite profiling analyses reveal the complex metabolic genes involved in the terpenoid biosynthesis in Blue Anise Sage (Salvia guaranitica L.). DNA Research , 25 (6), 597-617.

Ali, M., Li, P. H., She, G. B., Chen, D. F., Wan, X. C., \& Zhao, J. (2017). Transcriptome and metabolite analyses reveal the complex metabolic genes involved in volatile terpenoid biosynthesis in gardensage (Salvia officinalis ). Scientific Reports ,7 (1), 16074.

Barreto, L. C., Herken, D. M. D., Silva, B. M. R., MunneBosch, S., \& Garcia, Q. S. (2020). ABA and GA4 dynamic modulates secondary dormancy and germination in Syngonanthus verticillatus seeds.Planta, $251(4), 86$.

Cai, Y. P., Weng, K., Guo, Y., Peng, J., \& Zhu, Z. J. (2015). An integrated targeted metabolomic platform for high-throughput metabolite profiling and automated data processing. Metabolomics ,11 (6), 1575-1586.

Gong, C. S., Zhu, H. J., Lu, X. Q., Yang, D. D., Zhao, S. J., Umer, M. J., .. Liu, W. G. (2021). An integrated transcriptome and metabolome approach reveals the accumulation of taste-related metabolites and gene regulatory networks during watermelon fruit development. Planta, 254 (2), 35. 
Gong, T., Shu, D., Yang, J., Ding, Z. T., \& Tan, H. (2014). Sequencing and transcriptional analysis of the biosynthesis gene cluster of abscisic acid-producing Botrytis cinerea. International Journal of Molecular Sciences , 15 (10), 17396-17410.

Huang, J., Wang, K., Zuo, S., Chen, L., Ding, Z. Y. Elshazly M., \& Zhang, B. B. (2020). Unsaturated fatty acid promotes the production of triterpenoids in submerged fermentation of Sanghuangporus baumii .Food Bioscience, 37, 100712.

Kaori, M., Petr, T., Miho, M. K., Tomohiko, K., Shusei, S., Danuse, T., ... Tatsuo, K., 2006. Roles of Arabidopsis ATP/ADP isopentenyltransferases and tRNA isopentenyltransferases in cytokinin biosynthesis. PNAS , 103 (44), 16598-16603.

Kim, J., Lee, J. G., Hong, Y., \& Lee, E. J. (2019). Analysis of eight phytohormone concentrations, expression levels of ABA biosynthesis genes, and ripening-related transcription factors during fruit development in strawberry. Journal of Plant Physiology ,239, 52-60.

Kuhl, C., Tautenhahn, R., Bottcher, C., Larson, T. R., \& Neumann, S. (2012). Camera: an integrated strategy for compound spectra extraction and annotation of liquid chromatography/mass spectrometry data sets.Analytical Chemistry , 84 (1), 283-289.

Liu, Z. C., Sun, T. T., Wang, S. X., \& Zou, L. (2020). Cloning, molecular properties and differential expression analysis of the isopentenyl diphosphate isomerase gene in Sanghuangporus baumii .Biotechnology 83 Biotechnological Equipment, 34 (1), 623-630.

Liu, Z. C., Wang, S. X., Xu, X. R., Wang, S. T., Sun, T. T., \& Zou, L. (2021). Molecular cloning and characterization of a gene encoding HMG-CoA reductase involved in triterpenoids biosynthetic pathway from Sanghuangporus baumii . Biotechnology 83 Biotechnological Equipment, 35 (1), 796-804.

Livak, K. J., \& Schmittgen, T. D. (2001). Analysis of relative gene expression data using real-time quantitative PCR and the $2^{\text {-CT }}$ method. Methods, 25 (4), 402-408.

Meng, J., Wang, B., He, G., Wang, Y., Tang, X. F., Wang, S. M., .. Zhou, G. K. (2019). Metabolomics Integrated with Transcriptomics Reveals Redirection of the Phenylpropanoids Metabolic Flux in Ginkgo biloba . Journal of Agricultural and Food Chemistry,67 (11), 3284-3291.

Peter, O., Hiroyuki, T., Takanori, S., Katsuya, O., Hitoshi, O., \& Satoru, K. (2020). Paclobutrazol elevates auxin and abscisic acid, reduces gibberellins and zeatin and modulates their transporter genes in Marubakaido apple (Malus prunifolia Borkh. var. ringo Asami) rootstocks. Plant Physiology and Biochemistry , 155 , $502-511$.

Qian, Q., Yang, Y. H., Zhang, W. B., Hu, Y. L., Li, Y. G., Yu, H., \& Hou, X. L. (2021). A novel Arabidopsis gene RGAT1 is required for GA-mediated tapetum and pollen development. New Phytologist ,231 (1), $137-151$.

Ro, D. K., Paradise, E. M., Ouellet, M., Fisher, K. J., Newman, K. L., Ndungu, J. M., .. Keasling, J. D. (2006). Production of the antimalarial drug precursor artemisinic acid in engineered yeast.Nature, 440 (7086), 940-943.

Schmiderer, C., Grausgruber-Gröger, S., Grassi, P., Steinborn, R., \& Novak, J., 2010. Influence of gibberellin and daminozide on the expression of terpene synthases in common sage (Salvia officinalis ). Journal of Plant Physiology, 167 (10), 779-786.

Schomburg, F. M., Bizzell, C. M., Lee, D. J., Zeevaart, J. A. D., \& Amasino, R. M. (2003). Overexpression of a novel class of gibberellin 2-oxidases decreases gibberellin levels and creates dwarf plants.Plant Cell , 15 (1), 151-163.

Takei, K., Yamaya, T., \& Sakakibara, H. (2004). Arabidopsis CYP735A1 and CYP735A2 encode cytokinin 
hydroxylases that catalyze the biosynthesis of trans-Zeatin. Journal of Biological Chemistry ,279 (40), 4186641872.

Tan, B. C., Schwartz, S. H., Zeevaart, J. A., \& McCarty, D. R. (1997). Genetic control of abscisic acid biosynthesis in maize. PNAS ,94 (22), 12235-12240.

Wang, J. Q., Wen, X. F., Zhang, Y. Y., Zou, P. P., Cheng, L., Gan, R. Y., .. Geng, F. (2020). Quantitative proteomic and metabolomic analysis of Dictyophora indusiata fruiting bodies during post-harvest morphological development. Food Chemistry ,339 , 127884.

Zhang, D. H., Jiang, L. X., Li, N., Yu, X., Zhao, P., Li, T., \& Xu, J. W. (2017). Overexpression of the squalene epoxidase gene alone and in combination with the 3-hydroxy-3-methylglutaryl coenzyme a gene increases ganoderic acid production in Ganoderma lingzhi . Journal of Agricultural and Food Chemistry , 65 (23), 4683-4690.

Zhang, D., Ren, L., Yue, J. H., Wang, L., Zhuo, L. H., \& Shen, X. H. (2014). GA4 and IAA were involved in the morphogenesis and development of flowers in Agapanthus praecox ssp. orientalis. Journal of Plant Physiology , 171 (11), 966-976.

Zhang, Z. M., Tong, X., Peng, Y., Ma, P., Zhang, M. J., Lu, H. M., .. Liang, Y. Z. (2015). Multiscale peak detection in wavelet space. Analyst, 140 (23), 7955-7964.

Zheng, N., Ming, Y. F., Chu, J. Z., Yang, S. D., Wu, G. C., Li, W. H., .. Cheng, X. H., (2021). Optimization of Extraction Process and the Antioxidant Activity of Phenolics from Sanghuangporus baumii .Molecules, 26 (13), 3850.

\section{FIGURE LEGENDS}

FIGURE 1 The growth and development process of $S$. baumii

FIGURE 2 Metabolome analyses of different developmental stages of $S$. baumii, (A) PCA score plot of metabolite profiles of Myc, Pri and Fru stages of S. baumii, (B) Venn graph showing up- and downregulated DEMs from pairwise comparisons of Fru vs. Myc, Fru vs. Pri and Pri vs. Myc stages, (C) Bar graph of upand downregulated DEMs from pairwise comparisons, (D) Volcano map of up- and downregulated DEMs from pairwise comparisons, and (E) Hierarchical clustering of terpenoid-related DEMs in Myc, Pri and Fru stages

FIGURE 3 Transcriptome analyses of different developmental stages of $S$. baumii . (A) PCA score plot of transcript profiles from Myc, Pri and Fru stages, (B) Venn graph of up- and downregulated DEGs from pairwise comparisons of Fru vs. Myc, Fru vs. Pri and Pri vs. Myc stages, (C) Bar graph of up- and down regulated DEGs from pairwise comparisons, and (D) Correlation of DEGs and DEMs involved in terpenoid biosynthesis in S. baumii

FIGURE 4 Pathways and genes involved in the biosynthesis of terpenoids during the different developmental stages of $S$. baumii . The differential expression of each annotated gene is presented as a heatmap on the corresponding place of the gene with the scale ranging from white (low) to red (high); Square patterns with different colours represent the percentage of metabolites content in comparisons of different developmental stages; The pie chart patterns with different colours represent terpenoid hormone levels at different stages of development; Rectangles with different colours represent different terpenoid biosynthesis pathways

FIGURE 5 qRT-PCR validation of the expression levels of genes associated with terpenoid metabolism at the three developmental stages of $S$. baumii . qRT-PCR expression levels were calculated as a ratio relative to the level of expression in the Myc stage, which was set as 1

FIGURE 6 Differences between WT and IT S. baumii strains observed in the three developmental stages. (A) Differences in transcript levels of terpenoid biosynthetic genes between WT and ITS. baumii strains in the three developmental stages, (B) Differences in terpenoids contents between WT and IT S. baumiistrains 
in the three developmental stages, and (C) Differences in morphological characteristics between WT and IT $S$. baumii strains in the three developmental stages

FIGURE 7 Effects of four exogenous hormones on the growth and development of S. Baumii . (A) Effect of adding CZ on IT Myc growth, (B) Effects of four terpenoid hormones on growth rates of WT Myc and IT Myc, (C) Effects of TZ and GA4 on the growth of germinating mycelia in IT Pri, (D) Effects of four terpenoid hormones on the growth rate of germinating mycelia in WT Pri and IT Pri, (E) Effect of GA4 spraying on the growth of WT Fru, and (F) Effects of spraying four terpenoid hormones on dry weight of WT Fru and IT Fru

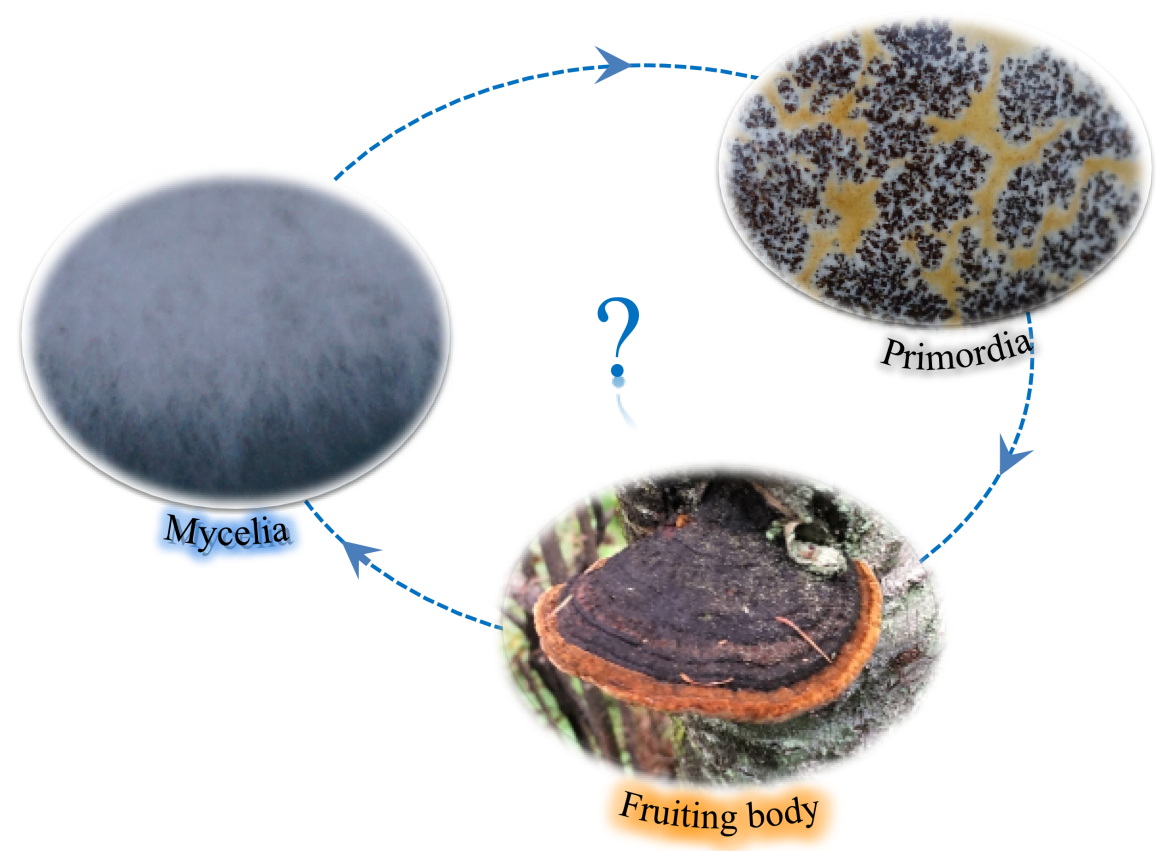



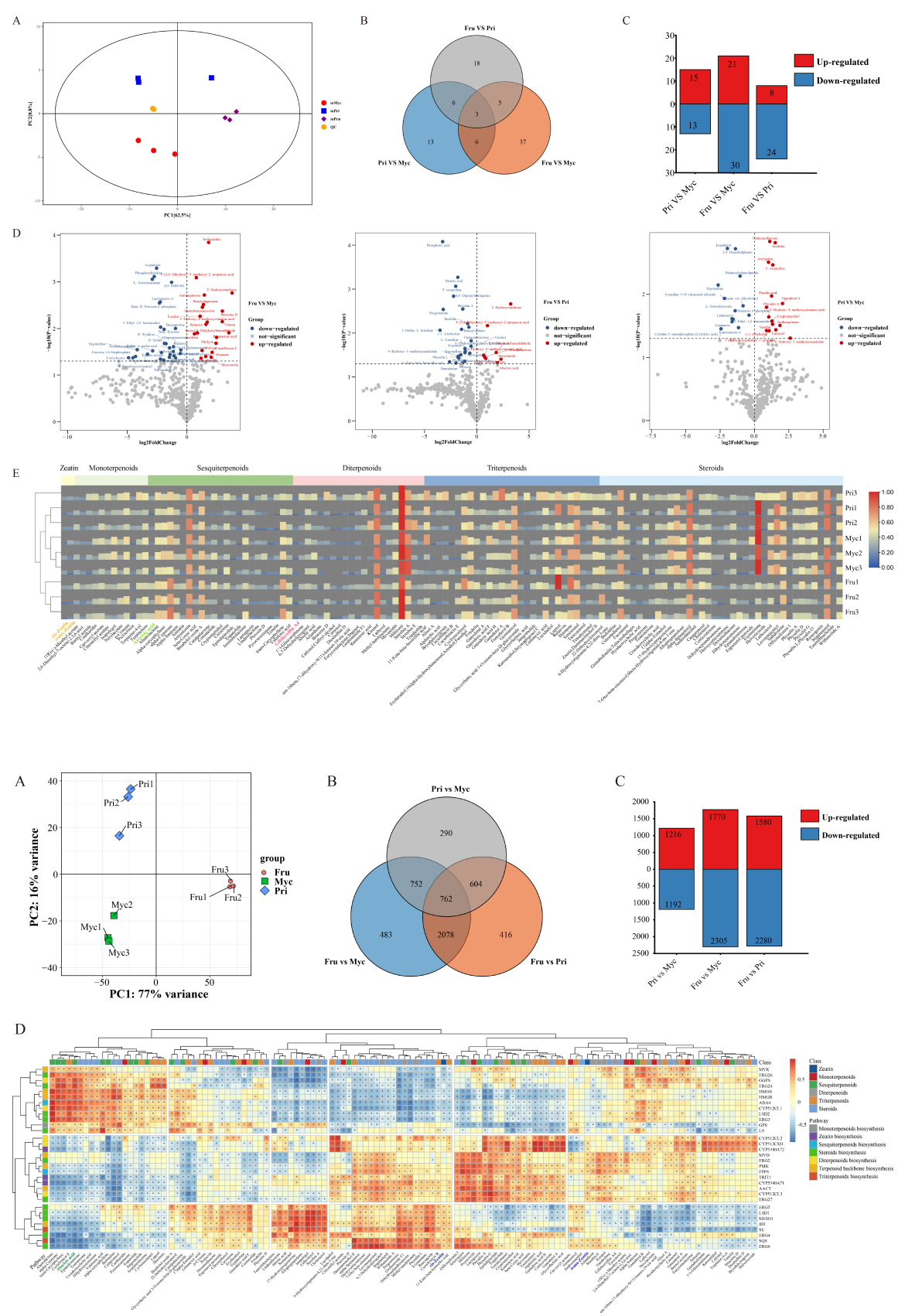

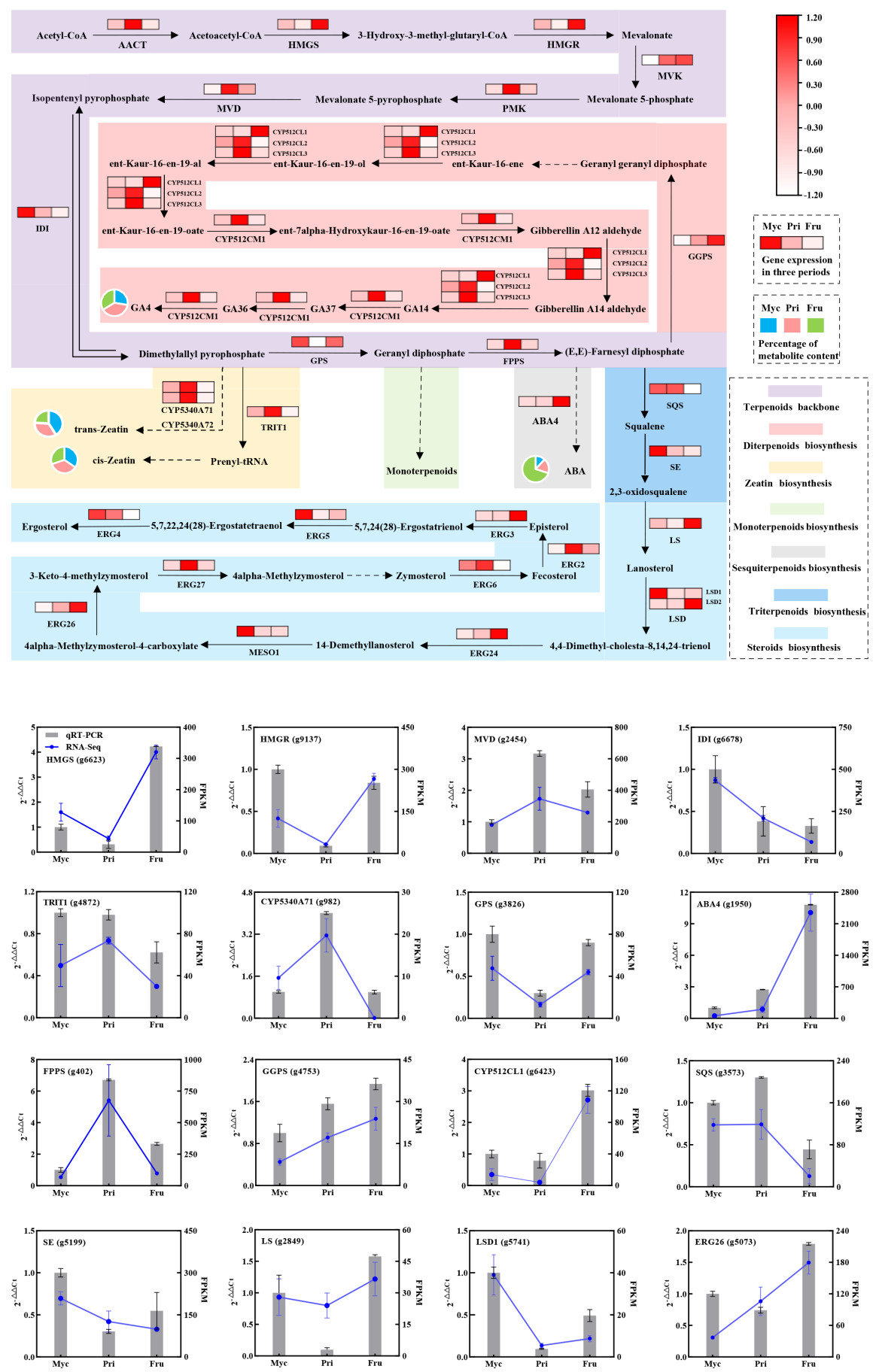

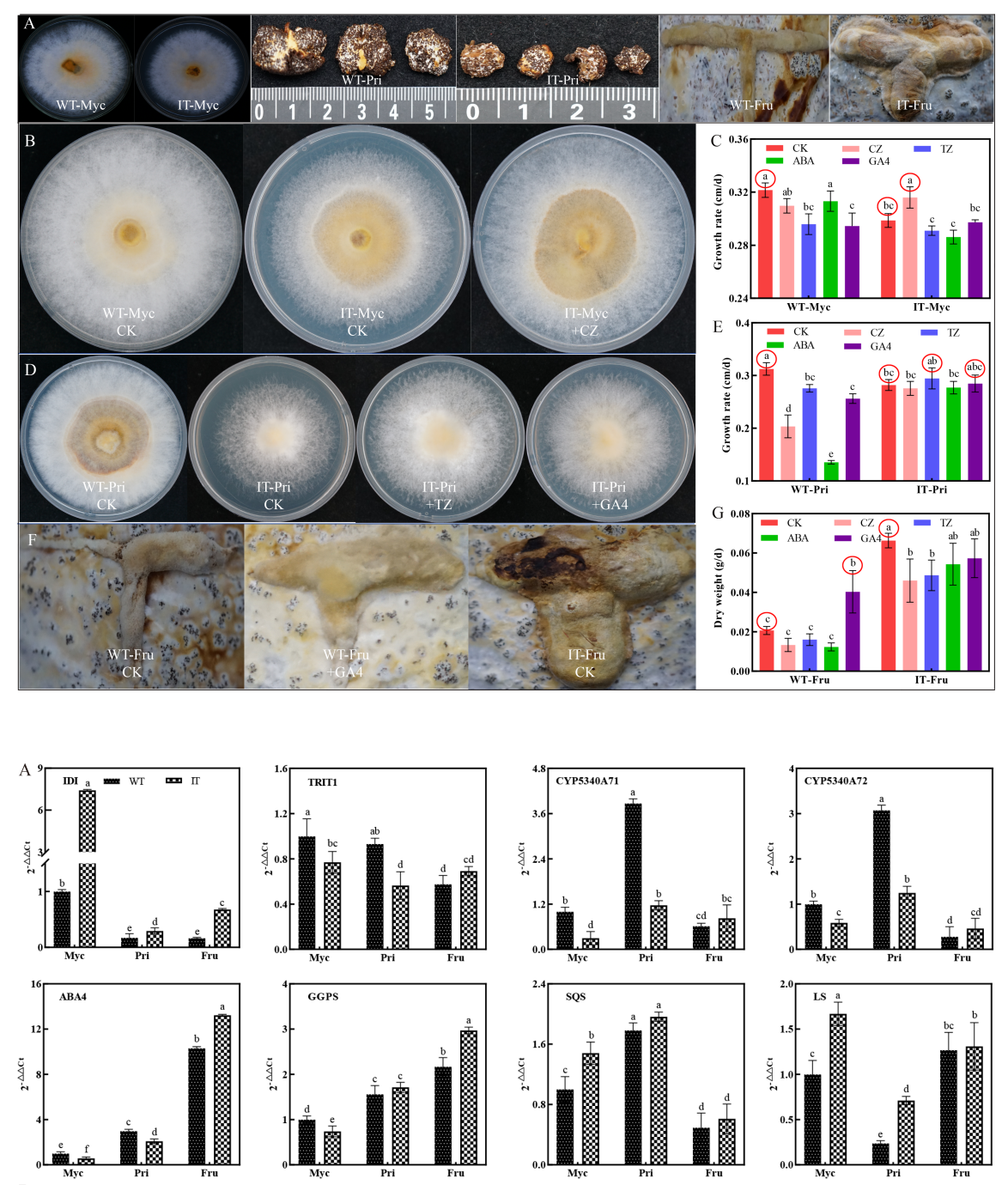

IIIIi
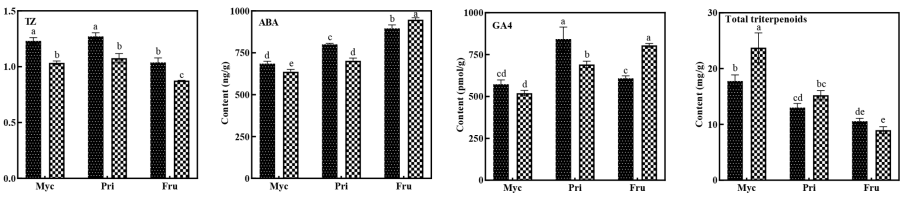